\title{
First draft genome of loach (Orenectus shuilongensis; Cypriniformes: Nemacheilidae) provide insights into the evolution of cavefish
}

\section{Zhijin Liu ( $\square$ liuzj@ioz.ac.cn )}

Kunming Institute of Zoology Chinese Academy of Sciences https://orcid.org/0000-0003-2923-1120

\section{Xuekun Qian}

Hebei University College of Life Sciences

\section{Ziming Wang}

Institute of Zoology

\section{Huamei Wen}

Guizhou Normal University School of Life Sciences

\section{Ling Han}

Guizhou Normal University School of Life Sciences

Jiang Zhou

Guizhou Normal University School of Life Sciences

\section{Research article}

Keywords: Oreonectes shuilongensis, cavefish, genome assembly, adaptation, Nemacheilidae, Cobitidea

Posted Date: February 5th, 2021

DOI: https://doi.org/10.21203/rs.3.rs-192229/v1

License: @ (1) This work is licensed under a Creative Commons Attribution 4.0 International License. Read Full License 


\section{Abstract}

Bcakground

Loaches of the superfamily Cobitoidea (Cypriniformes, Nemacheilidae) are small elongated bottom-dwelling freshwater fishes with several barbels near the mouth. The genus Oreonectes with 18 currently recognized species contains representatives for all three key stages of the evolutionary process (a surface-dwelling lifestyle, facultative cave persistence, and permanent cave dwelling). Some Oreonectes species show typical cave dwellingrelated traits, such as partial or complete leucism and regression of the eyes, rendering them as suitable study objects of micro-evolution. Genome information of Oreonectes species is therefore an indispensable resource for research into the evolution of cavefishes.

Results

Here we assembled the genome sequence of $O$. shuilongensis, a surface-dwelling species, using an integrated approach that combined PacBio single-molecule real-time sequencing and Illumina $\mathrm{X}$-ten paired-end sequencing. Based on in total $50.9 \mathrm{~Gb}$ of sequencing data, our genome assembly from Canu and Pilon spans approximately $515.64 \mathrm{Mb}$ (estimated coverage of $100 \times$ ), containing 803 contigs with N50 values of $5.58 \mathrm{Mb}$. 25,247 proteincoding genes were predicted, of which $95.65 \%$ have been functionally annotated. We also performed genome resequencing of three additional cave-dwelling Oreonectes fishes. Twenty-nine pseudogenes annotated using DAVID showed significant enrichment for the GO terms of "eye development" and "retina development in camera-type eye". It is presumed that these pseudogenes might lead to eye degeneration of semi/complete cave-dwelling Oreonectes species. Furthermore, Mc1r (melanocortin-1 receptor) is a pseudogenization by a deletion in 0 . daqikongensis, likely blocking biosynthesis of melanin and leading to the albino phenotype.

\section{Conclusions}

We here report the first draft genome assembly of Oreonectes fishes, which is also the first genome reference for Cobitidea fishes. Pseudogenization of genes related to body color and eye development may be responsible for loss of pigmentation and vision deterioration in cave-dwelling species. This genome assembly will contribute to the study of the evolution and adaptation of fishes within Oreonectes and beyond (Cobitidea).

\section{Background}

Cavefishes are successful vertebrate colonizers in subterranean habitats and usually process some regressive features, such as the rudimentary eyes and loss of pigmentation. Meanwhile, some compensative traits, such as elongated appendages and reinforced non-visual sensory systems, have evolved in cavefishes. Since uncovering the genetic basis of phenotypic adaptations of animals to a specific environment is a key goal in evolutionary study, cavefishes have attracted interests from biologist and certain cavefishes (Astyanax mexicanus and Sinocyclocheilus spp.) have been well studied. However, there is few data available to unravel the genomic mechanism under the evolution and adaptation to subterranean life among other groups of fishes.

Loaches (Cypriniformes: Cobitoidea, Nemacheilidae) are small elongated bottom-dwelling freshwater fishes with several barbels near the mouth, distributed in Eurasia and Africa. Within this group, Oreonectes fishes are distributed only in southwestern China and northern Vietnam, most of which dwell in underground rivers in the karst environment ${ }^{[1]}$. Oreonectes fishes contain 18 species representing the three key stages of the evolutionary 
process including a surface-dwelling lifestyle, facultative cave persistence, and permanent cave dwelling. Almost all Oreonectes species show some cave-related traits, such as part or complete eye degeneration and leucism, which makes this genus a good study system of micro-evolution ${ }^{[2]}$. O. shuilongensis is a surface-dwelling species which was newly discovered in the Shuilong Township in Guizhou Province of China ${ }^{[3]}$. A genome assembly of $O$. shuilongensis would facilitate research into key aspects of the evolutionary history of cave versus surface dwelling in Oreonectes, including the role of environmental changes in the seeminly rapid diversification and speciation in underground caves ${ }^{[2]}$.

Here we present a de novo genome assembly for 0 . shuilongensis, the first genome constructed for the family Nemacheilidae and the superfamily Cobitidea. The completeness and continuity of the genome provided valuable genomic resources for studies on the evolutionary history of the rapid speciation processes of family Nemacheilidae.

\section{Results}

\section{Whole Genome and RNA Sequencing}

After removal of < 500 bp PacBio subreads, 5 million subreads (total $50.9 \mathrm{~Gb}$ ) remained, with an average length of $10.2 \mathrm{~kb}$ (Table 1 and Suppl. Tables S2 and S3). Additionally, a total of $11.7 \mathrm{~Gb}$ transcriptome data were obtained from RNA-sequencing (Table 1).

Table 1

Summary of sequence data from Orenectus shuilongensis. The sequencing data used in this work. Note that read length for PacBio Sequel were measured for subreads.

\begin{tabular}{|llllll|}
\hline Type & Libraries & $\begin{array}{l}\text { Insert size } \\
(\mathbf{b p})\end{array}$ & $\begin{array}{l}\text { Read length } \\
(\mathbf{b p})\end{array}$ & $\begin{array}{l}\text { Clean data } \\
(\mathbf{G b})\end{array}$ & $\begin{array}{l}\text { Coverage } \\
(\mathbf{X})\end{array}$ \\
\hline DNA & HiSeq X Ten & 270 & 150 & 120.94 & 234.55 \\
\hline DNA & PacBio & 20,000 & 10,187 & 50.94 & 98.79 \\
\hline RNA & HiSeq X Ten & 270 & 150 & 11.7 & - \\
\hline The coverage was calculated using an estimated genome size of 515.64 Mb.
\end{tabular}

\section{Estimation of the Genome Size and Sequencing Coverage}

The genome size of 0 . shuilongensis was estimated at approximately $515.66 \mathrm{Mb}$ based on k-mer analysis (Suppl. Figure S1), and our $O$. shuilongensis genome assembly spans $521.68 \mathrm{Mb}$ (803 contigs, contig N50 of $5.58 \mathrm{Mb}$; Table 2 and Suppl. Table S4). The completeness of the 0 . shuilongensis genome assembly was evaluated using CEGMA v2.5 $5^{[4]}$ and BUSCO v2 ${ }^{[5]}$. CEGMA analysis suggested that $99.78 \%$ of conserved Core Eukaryotic Genes (CGEs) proteins are present in our assembled genome, and BUSCO analysis showed that $97.58 \%$ of vertebrate Benchmarking Universal Single-Copy Orthologs have been assembled, implying a high completeness of our 0 . shuilongensis genome assembly (Suppl. Tables S5 and S6). We found around $99.52 \%$ of the reads properly mapped to the genome assembly (Suppl. Table S7). All these results indicate that the assembly of the 0 . shuilongensis genome is characterized by a high level of accuracy. 
Table 2

Summary of the loach (O. shuilongensis) genome assembly and annotation.

\begin{tabular}{|ll|}
\hline Genome quality & values \\
\hline Contig N50 size $(\mathrm{Mb})$ & 5.58 \\
\hline Estimated genome size $(\mathrm{Mb})$ & 515.64 \\
\hline Assembled genome size (Mb) & 521.68 \\
\hline Genome coverage (fold) & $234.55 \times$ \\
\hline Content of transposable elements (\%) & 23.42 \\
\hline Total GC content (\%) & 38.51 \\
\hline Protein-coding gene numbers & 25,247 \\
\hline Average gene length (kb) & 234.62 \\
\hline Average CDS length (bp) & 2,010 \\
\hline
\end{tabular}

\section{Annotation of Repeat Sequences, Protein Coding Genes and Noncoding RNA}

We found that repetitive elements comprised $23.42 \%$ of the 0 . shuilongensis genome (Table 3 ). 25,247 proteincoding genes were identified. The average transcript length, CDS length, and intron length were 9,744 bp, 2,010 bp, and 7,734 bp, respectively (Suppl. Table S8). Among these annotated genes, $64.51 \%$ of encoded proteins showed homology to proteins in the KOG database, $95.53 \%$ were identified in the NCBI non-redundant database, $46.72 \%$ were identified in the KEGG database, 94.90\% were identified in the TrEMBL database, and 95.65\% could be mapped onto the functional databases (Suppl. Table S9). Finally, 947 miRNAs, 561 rRNAs and 417 tRNAs were discovered from the 0 . shuilongensis (Suppl. Table S10). 
Table 3

Annotation of repeat sequences in the loach (O. shuilongensis) genome assembly.

\begin{tabular}{|c|c|c|c|}
\hline Type & Number & Length(bp) & Percentage (\%) \\
\hline ClassI/DIRS & 1,566 & $1,563,184$ & 0.3 \\
\hline ClassI/LINE & 6,300 & $11,594,987$ & 2.22 \\
\hline Classl/LTR & 5,947 & $4,877,346$ & 0.93 \\
\hline ClassI/LTR/Copia & 909 & 528,739 & 0.1 \\
\hline ClassI/LTR/Gypsy & 9,340 & $6,473,497$ & 1.24 \\
\hline ClassI/PLE|LARD & 7,579 & $15,588,001$ & 2.99 \\
\hline ClassI/SINE & 4,322 & 591,812 & 0.11 \\
\hline Classl/TRIM & 3,358 & $3,025,034$ & 0.58 \\
\hline Classl/Unknown & 342 & 35,272 & 0.01 \\
\hline ClassII/Crypton & 11,782 & $2,048,568$ & 0.39 \\
\hline ClassII/Helitron & 8,994 & $1,095,311$ & 0.21 \\
\hline Classll/MITE & 4,176 & 596,316 & 0.11 \\
\hline Classll/Maverick & 2,145 & 374,478 & 0.07 \\
\hline ClassII/TIR & 71,957 & $21,304,786$ & 4.08 \\
\hline Classll/Unknown & 178,045 & $22,476,697$ & 4.31 \\
\hline PotentialHostGene & 32,601 & $4,535,251$ & 0.87 \\
\hline SSR & 797 & 112,798 & 0.02 \\
\hline Unknown & 178,496 & $25,369,612$ & 4.86 \\
\hline Total without overlap: & 528,656 & $122,191,689$ & 23.42 \\
\hline
\end{tabular}

\section{Phylogenetic relationship and genomic comparison}

As a result, 16,708 gene families were constructed for the 0 . shuilongensis. Among the families, there were 144 families unique to 0 . shuilongensis (Fig. 2 and Suppl. Table S11). The constructed phylogenetic tree indicated that O. shuilongensis were clustered closely to Cyprinidae species, which is inconsistent with their putative evolutionary relationships (Fig. 3). The divergence between 0 . shuilongensis and cyprinid fishes (Cyprinidae) occurred ca. 91.31 million years ago (95\% HPD, 82.58-108.26). When comparing this with the other seven fish (S. salar, I. punctatus, A. mexicanus, $C$. carpio, S. rhinocerous, D. rerio and $L$. crocea), the expansion and contraction of gene ortholog clusters showed 77 gene families were expanded and 282 gene families contracted significantly in the 0 . shuilongensis (Fig. 3).

\section{Genomic mechanism underlying the degeneration of eyes and body color}


Genome re-sequencing were performed for facultative cave-dwelling 0 . jiarongensis (three individuals) and cavedwelling 0 . daqikongensis (two individuals) and 0 . dongliangensis (one individual) at a high average depth of $28.06 \pm 5.08 \times$, with an overall average genome coverage of $93.77 \%$ of the 0 . shuilongensis genome assembly (Table 4). A total of 12,534,348 SNPs was identified in these three species, and the number of SNPs per individual ranged from 6.0 to $6.2 \mathrm{M}$ (Table 4). The reconstructed phylogeny indicates that the ancestor of cave-dwelling 0 . daqikongensis, 0 . dongliangensis and facultative cave-dwelling 0 . jiarongensis first diverged from the surfacedwelling O. shuilongensis about 9.31 million years ago (95\% HPD, 12.54-7.12) (Fig. 4).

Table 4

Re-sequencing analysis of cave-dwelling Orenectus fishes.

\begin{tabular}{|c|c|c|c|c|c|c|}
\hline \multirow{2}{*}{$\begin{array}{l}\text { Species } \\
\text { Sample ID }\end{array}$} & \multicolumn{3}{|c|}{ O. jiarongensis } & \multicolumn{2}{|c|}{ O. daqikongensis } & \multirow{2}{*}{$\begin{array}{l}\text { O. } \\
\text { dongliangensis } \\
\text { R06 }\end{array}$} \\
\hline & R01 & R02 & R03 & R04 & R05 & \\
\hline Total_reads & 154919048 & 145879318 & 136865354 & 147160730 & 126896868 & 123201446 \\
\hline Clean_Reads & 77459524 & 72939659 & 68432677 & 73580365 & 63448434 & 61600723 \\
\hline $\mathrm{GC}(\%)$ & 39.06 & 39.02 & 38.92 & 38.92 & 38.95 & 39.38 \\
\hline Mapping_rate & $90.49 \%$ & $91.15 \%$ & $91.83 \%$ & $90.82 \%$ & $91.63 \%$ & $92.26 \%$ \\
\hline Ave_depth & 29 & 28 & 26 & 30 & 26 & 24 \\
\hline Cov_ratio_1X(\%) & 87.44 & 87.76 & 87.62 & 87.26 & 86.98 & 87.46 \\
\hline Cov_ratio_5X(\%) & 83.46 & 83.74 & 83.5 & 83.37 & 82.76 & 83.01 \\
\hline Cov_ratio_10X(\%) & 80.35 & 80.56 & 80.07 & 80.44 & 79.3 & 79.05 \\
\hline Total_SNP & 6201592 & 6207932 & 6201733 & 6064030 & 6049874 & 6189968 \\
\hline Transition & 3544204 & 3547586 & 3544063 & 3485910 & 3478106 & 3539101 \\
\hline Transversion & 2657388 & 2660346 & 2657670 & 2578120 & 2571768 & 2650867 \\
\hline $\mathrm{Ti} / \mathrm{Tv}$ & 1.33 & 1.33 & 1.33 & 1.35 & 1.35 & 1.33 \\
\hline Het_snp & 199131 & 193488 & 191382 & 183450 & 182330 & 248330 \\
\hline Hom_snp & 6002461 & 6014444 & 6010351 & 5880580 & 5867544 & 5941638 \\
\hline Het-ratio & $3.21 \%$ & $3.11 \%$ & $3.08 \%$ & $3.02 \%$ & $3.01 \%$ & $4.01 \%$ \\
\hline Total_Indel & 2335694 & 2340858 & 2336570 & 2218308 & 2208883 & 2321897 \\
\hline Het_Indel & 113531 & 111329 & 108840 & 112158 & 110204 & 135160 \\
\hline Hom_Indel & 2222163 & 2229529 & 2227730 & 2106150 & 2098679 & 2186737 \\
\hline
\end{tabular}

Annotation of all sequence variants in $\operatorname{SnpEff}^{[6]}$ (Fig. 5) suggested that 1,541 SNPs and 438 indels were located in 401 genes, likely resulting in pseudogenization in semi cave-dwelling and cave-dwelling species. Twenty-nine 
pseudogenes annotated using DAVID ${ }^{[7]}$ showed significant enrichment for the GO terms of "eye development (0001654)" and "retina development in camera-type eye (0060041)" (Table 5, Fig. 6 and Suppl. Table S12). For example, the expression of $\operatorname{six} 7$, six $6 a$ and six $6 b$ is required for optic primordium development and the specification and proliferation of the eye field in vertebrate embryos ${ }^{[8]}$. The function-lost mutation of $a / d h 1 a 3$ and tfap2a caused eye and retinal defects in zebrafish ${ }^{[9]}$. It is presumed that these pseudogenes might lead to eye degeneration of semi/complete cave-dwelling Oreonectes species. Furthermore, Mc1r (melanocortin-1 receptor), a key gene regulating the biosynthesis of melanin in most vertebrates, is a pseudogenization caused by a deletion in O. daqikongensis (Fig. 7), likely blocking biosynthesis of melanin and leading to the albino phenotype (Fig. 1). Remaining pseudogenes are enriched for the GO terms of "potassium channel activity", "regulation of axon extension involved in axon guidance”, "G-protein coupled receptor activity" and KEGG pathway of "neuroactive ligand-receptor interaction” (Table 5). 
Table 5

Functional classification and enrichment analysis of pseudogenes in cave-dwelling Oreonectes fishes. Gray boxed GOs are associated with retina and eye development.

\begin{tabular}{|c|c|c|c|c|}
\hline GO_ID & GO_Term & $\begin{array}{l}\text { Go } \\
\text { Class }\end{array}$ & $\begin{array}{l}\text { Gene } \\
\text { num }\end{array}$ & $\begin{array}{l}\text { Adjusted } P \\
\text { value }\end{array}$ \\
\hline G0:0060041 & retina development in camera-type eye & $\mathrm{BP}$ & 9 & 2.8E-9 \\
\hline G0:0001654 & eye development & $\mathrm{BP}$ & 8 & 8.9E-9 \\
\hline GO:0051260 & protein homooligomerization & $\mathrm{BP}$ & 4 & 0.0031 \\
\hline G0:0007186 & G-protein coupled receptor signaling pathway & $\mathrm{BP}$ & 11 & 0.0033 \\
\hline G0:0007165 & signal transduction & $\mathrm{BP}$ & 13 & 0.0043 \\
\hline G0:0034765 & regulation of ion transmembrane transport & $\mathrm{BP}$ & 4 & 0.0085 \\
\hline G0:0006813 & potassium ion transport & $\mathrm{BP}$ & 4 & 0.013 \\
\hline G0:0071805 & potassium ion transmembrane transport & $\mathrm{BP}$ & 5 & 0.013 \\
\hline G0:0021785 & branchiomotor neuron axon guidance & $\mathrm{BP}$ & 2 & 0.025 \\
\hline G0:0048841 & regulation of axon extension involved in axon guidance & $\mathrm{BP}$ & 2 & 0.033 \\
\hline G0:1902287 & $\begin{array}{l}\text { semaphorin-plexin signaling pathway involved in axon } \\
\text { guidance }\end{array}$ & $\mathrm{BP}$ & 2 & 0.033 \\
\hline G0:0005267 & potassium channel activity & MF & 5 & 0.00012 \\
\hline G0:0004930 & G-protein coupled receptor activity & MF & 10 & 0.0083 \\
\hline GO:0005251 & delayed rectifier potassium channel activity & MF & 3 & 0.011 \\
\hline G0:0005244 & voltage-gated ion channel activity & MF & 4 & 0.0038 \\
\hline G0:0005249 & voltage-gated potassium channel activity & MF & 4 & 0.0038 \\
\hline G0:0046332 & SMAD binding & MF & 2 & 0.044 \\
\hline G0:0008076 & voltage-gated potassium channel complex & $\mathrm{CC}$ & 4 & 0.0038 \\
\hline G0:0002116 & semaphorin receptor complex & $\mathrm{CC}$ & 2 & 0.075 \\
\hline G0:0000276 & $\begin{array}{l}\text { mitochondrial proton-transporting ATP synthase complex, } \\
\text { coupling factor }\end{array}$ & $\mathrm{CC}$ & 2 & 0.049 \\
\hline dre04080* & Neuroactive ligand-receptor interaction & KEGG & 5 & 0.0088 \\
\hline
\end{tabular}

\section{Discussion And Conclusions}

Organisms that have colonized underground caves encounter vastly different selective pressures than their relatives in above-ground habitats. In the present study, we report the first whole genome sequencing, assembly, and annotation of the 0 . shuilongensis, encompassing a total of predicted 25,247 protein-coding genes and 7,041 noncoding RNAs. We anticipate that this genome assembly will serve as a basis for in-depth biological studies of evolution and adaptation of cavefishes. With the availability of these genomic data, genomic/transcriptomics 
differences between surface-dwelling and cave-dwelling loaches can be studied at the genomic scale. More broadly, our assembly will facilitate evolutionary and genomics research of Cobitoidea fishes and beyond.

\section{Materials And Methods}

\section{Animals captured and DNA extracted}

O. shuilongensis, O. jiarongensis (three individuals), O. dongliangensis (two individuals) and O. daqikongensis (one individuals) (Fig. 1) were captured from Guizhou Province, China. Each individual was over-exposed and executed by anesthesia, and quickly frozen in liquid nitrogen for one hour before storing at $-80^{\circ} \mathrm{C}$. Genomic DNA was extracted from a muscle sample using DNeasy Blood \&Tissue Kit (Qiagen). The present study was approved by the Animal Ethics Committee of Guizhou Normal University. The procedure of sample collection was in strict accordance with the Animal Ethics Procedures and Guidelines of the People's Republic of China.

\section{Whole Genome Sequencing of O. slonglongensis}

Three small-insert libraries (270 bp) were constructed by using Illumina's paired-end kits according to the manufacturer's instructions. The libraries were sequenced on Illumina Hiseq X Ten platform. For the raw reads, sequencing adaptors were removed. Contaminated reads (such as chloroplast, mitochondrial, bacterial and viral sequences, etc.) were screened by alignment to the NCBI-NR database using BWA ${ }^{[10]}$ with default parameters. Duplicated read pairs were removed by FastUniq $v 1.12^{[11]}$, and low-quality reads were filtered under the following conditions: (1) reads with $\geq 10 \%$ unidentified nucleotides $(N),(2)$ reads with $>10$ nucleotides aligned to the adapter, allowing $\leq 10 \%$ mismatches, (3) reads with $>50 \%$ bases having Phred quality $<5$.

\section{RNA Sequencing}

Tissues of skin, muscle, intestine, liver and kidney of the same loach individual were collected and RNAs were extracted with TRIZOL Reagent (Invitrogen, USA). RNAs were then balanced mixed for the sequencing. The absorbance of 1.90 at $260 \mathrm{~nm} / 280 \mathrm{~nm}$ and the RIN of 9.1 were obtained for the purified RNA sample by Nanodrop ND-1000 spectrophotometer (LabTech, USA) and 2100 Bioanalyzer (Agilent Technologies, USA), respectively. One microgram of RNA was reverse transcribed using Clontech SMARTer cDNA synthesis kit, and was further fragmented using divalent cations for the sequencing. The paired-end library was prepared following the manual of the Paired-End Sample Preparation Kit (Illumina Inc., San Diego, CA, USA). Then the library with an insert length of 270 bp was sequenced by Illumina HiSeq X Ten in 150 bp paired-end mode (Illumina Inc., San Diego, CA, USA).

\section{Estimation of the Genome size and Sequencing Coverage}

The 19-mer frequency distribution analysis was performed on the remaining clean reads to estimate the genome size of the 0 . shuilongensis using the formula: Genome size= kmer_Number/Peak_Depth. Corrected Illumina reads were selected to perform genome size estimation.

\section{De novo Genome Assembly and Quality Assessment of 0. shuilongensis Genome}

The single-molecule sequencing (SMS) data are assembled through Canu v1.5 ${ }^{[12]}$ a comprehensive and scalable pipeline for SMS data assembly (https://github.com/marbl/canu), and the draft assembly polished through Pilon ${ }^{[13]}$. In the correction step, we used Canu to first select longer seed reads with the settings 'genomeSize = 
$520 \mathrm{M}$ ' and 'corOutCoverage $=90$ ', then to detect raw reads overlapping through a highly sensitive over lapper MHAP (mhap-2.1.2, option 'corMhapSensitivity = normal'), and finally to perform error correction through the falcon sense method (option 'corrected Error Rate $=0.045$ '). Next, using default parameter settings, error-corrected reads were trimmed of unsupported bases and hairpin adapters to obtain their longest supported range. In the last step, Canu generated the draft assembly using trimmed reads. The draft assembly was polished to obtain the final assembly, adopting Pilon ${ }^{[13]}$ (https://github.com/broadinstitute/pilon) using Illumina data with the parameters 'mindepth 10 -changes --threads 4 -fix bases'. To further evaluate the accuracy of the 0 . shuilongensis genome, we aligned the high-quality short reads from short insert size pair-ended libraries against the genome assembly using BWA $^{[14]}$

\section{Annotation of Repeat Sequences}

The repeat composition of the assemblies was estimated by building a repeat library employing the de novo prediction programs LTR FINDER v1.05 ${ }^{[15]}$, MITE-Hunter ${ }^{[16]}$, RepeatScout v1.0.5 ${ }^{[17]}$ and PILER-DF v2.4 ${ }^{[18]}$. The database was classified using PASTEClassifier ${ }^{[19]}$ and was combined with the Repbase ${ }^{[20]}$ database to create the final repeat library. Repeat sequences in the $O$. shuilongensis genome were identified and classified using the RepeatMasker v4.0.6 ${ }^{[21]}$ program.

\section{Annotation of Protein Coding Genes}

Protein-coding genes were predicted based on de novo, protein homology-based and RNA-Seq method. We first used five de novo gene prediction tools including Genscan ${ }^{[22]}$, Augustus v2.4 ${ }^{[23]}$, GlimmerHMM v3.0.4 ${ }^{[24]}$, GenelD $\mathrm{v} 1.4^{[25]}$ and SNAP ${ }^{[12]}$ to predict protein-coding genes in the 0 . shuilongensis genome. For homology-based gene prediction, protein sequences from four closely related teleost species including Cyprinus carpio, Danio rerio, Sinocyclocheilus rhinocerous and Astyanax mexicanus were downloaded from Ensembl database and aligned against to the 0 . shuilongensis genome using GeMoMa v1.3.1 ${ }^{[26]}$ software; the RNA-Seq reads were assembled into contigs de novo into unigenes using Trinity and the unigenes were aligned to the repeat-masked assemblies using BLAT ${ }^{[27]}$, and subsequently the gene structures of BLAT alignment results were modeled using PASA v2.02 ${ }^{[28]}$. Additionally, the RNA-Seq reads were also assembled into transcripts through mapping to the assembled genome using Hisat2 v2.0.4 ${ }^{[29]}$ and StringTie v1.3.0 ${ }^{[30]}$, and the protein-coding regions were identified with TransDecoder v2.0 ${ }^{[31]}$ and GeneMarkS-T v5. ${ }^{[32]}$, respectively. All these consensus gene models were generated by integrating the de novo predictions, protein alignments and transcripts data using EVidenceModeler ${ }^{[33]}$.

\section{Gene Functional Annotation}

Annotation of the predicted genes was performed by local BLAST ${ }^{[27]}$ programs blasting their sequences against a number of nucleotide and protein sequence databases, including NCBI-NR ${ }^{[20]}, \mathrm{KOG}^{[34]}, \mathrm{KEGG}^{[35]}$, and $\operatorname{TrEMBL}^{[36]}$ with an E-value cutoff of 1e-5. We then searched the Gene ontology (GO) and Kyoto Encyclopedia of Genes and Genomes (KEGG) pathway databases using the software Blast2GO ${ }^{[37]}$.

\section{Noncoding RNA Annotation}

Non-coding RNAs play important roles in a great variety of processes, such as the rRNAs and tRNAs involved in mRNA translation. The rRNA fragments were identified by aligning the rRNA template sequences using BLAST with E-value at 1 e-10 and identity cutoff at $95 \%$ or more. The tRNAScan-SE v1.3.1 ${ }^{[38]}$ algorithms with default parameters were applied to the prediction of tRNA genes. The rRNA and miRNA genes were predicted by Infenal 
$\mathrm{v} 1.1^{[39]}$ against the Rfam ${ }^{[40]}$ database and miRbase ${ }^{[41]}$ with cutoff score at 30 or more. The minimum a cutoff score was based on the settings which yield a false positive rate of 30 bits.

\section{Global Gene Family Classification}

In order to identify gene families among fish species in this work, proteins of the longest transcripts of each individual gene from $O$. shuilongensis and other sequenced species, including Salmo salar, Ictalurus punctatus, $A$. mexicanus, $C$. carpio, S. rhinocerous, $D$. rerio and Larimichthys crocea were analyzed. All data was downloaded from NCBI ${ }^{[42]}$. Gene family analysis based on the homolog of gene sequences in related species was initially implemented by the alignment of an "all against all" BLASTP ${ }^{[43]}$ with a cutoff of 1 e- 5 and subsequently followed by alignments with high-scoring segment pairs conjoined for each gene pair by Solar. To identify homologous gene pairs, we required more than $30 \%$ coverage of the aligned regions in both homologous genes. Finally, homologous genes were clustered into gene families by OrthoMCL ${ }^{[44]}$ with the inflation parameter set at 1.5 .

\section{Phylogenetic Relationship and Genomic Comparison}

Evolutionary analysis was performed using the single-copy protein-coding genes among all species. Amino acid and nucleotide sequences of the ortholog genes were aligned using the multiple alignment software MUSCLE ${ }^{[18]}$ with default parameters. A total number of 724 single-copy ortholog alignments were concatenated into a super alignment matrix of 1,692,085 nucleotides. A maximum likelihood method deduced tree was inferred based on the matrix of nucleotide sequences using PhyML package with the JTT + G + F model. Clade support was assessed using bootstrapping algorithm in the PhyML package with 100 alignment replicates. The constructed phylogenetic tree indicated that $O$. shuilongensis were clustered closely to Cyprinidae species, which is inconsistent with their putative evolutionary relationships (Fig. 3).

We determined the expansion and contraction of the orthologous gene families by comparing the cluster size differences between the ancestor and each of the 0 . shuilongensis and seven other fish species using the CAFÉ[45] program. A random birth and death model were used to study changes of gene families along each lineage of the phylogenetic tree. A probabilistic graphical model (PGM) was introduced to calculate the probability of transitions in gene family size from parent to child nodes in the phylogeny. Using conditional likelihoods as the test statistics, we calculated the corresponding $P$-values in each lineage. A $P$-value of 0.05 was used to identify families that were significantly expanded in 0 . shuilongensis genome.

\section{Dating the Divergence among Oreonectes Fishes and Assessing the Genomic Mechanism Underlying the Degeneration of Eyes and Body Color}

O. shuilongensis is a surface-dwelling species with intact eyes and a unique color pattern consisting of fine black marks on the body except on the abdomen ${ }^{[3]}$. To explore the genomic changes resulting in the degeneration of eyes and body color of semi cave-dwelling and cave-dwelling Oreonectes fishes, the whole genome re-sequencing was performed for facultative cave-dwelling 0 . jiarongensis (three individuals) and cave-dwelling 0 . daqikongensis (two individuals) and $O$. dongliangensis (one individual). For each individual, $\sim 3 \mu \mathrm{g}$ of DNA was sheared into fragments of $350 \mathrm{bp}$ with the Covaris v1.8 system. DNA fragments were then processed and sequenced using the Illumina HiSeq 4000 platform. Filtered sequence reads were mapped to the $O$. shuilongensis reference genome using BWA-MEM with default parameters (0.7.10-r789) ${ }^{[46]}$. Alignment bam files were imported to SAMtools (v0.1.19) ${ }^{[10]}$ for sorting and Picard (http://broadinstitute.github.io/picard/, version 1.92) was used to remove duplicated reads. Following mapping, we performed variant calling using the GATK $^{[47]}$ package with default 
parameters. To explore the phylogenetic relationships among Oreonectes fishes, phylogenetic tree was inferred using the Neighbor-Joining (NJ) algorithm as implemented in RAxML software with 1000 bootstraps ${ }^{[48]}$ and the divergence times of the taxa analyzed were estimated with mcmctree ${ }^{[15]}$. The outgroup sequences were chosen from the zebrafish genome assembly GRCz11, with the genome alignment to the obtained Oreonectes genome assembly using LASTZ ${ }^{[49]}$. We employed calibration points from the dated Cyprinidae-Nemacheilidae divergence to place a lognormally distributed prior on the age of the root of a tree containing all samples of Oreonectes species and outgroup. To explore the genomic mechanism underlying the degeneration of eyes and body color, the SNPs and Indels obtained through GATK pipeline were annotated using the software SnpEff ${ }^{[6]}$.

\section{Abbreviations}

BLAST: Basic Local Alignment Search Tool; KEGG: Kyoto Encyclopedia of Genes and Genomes; NCBI: National Center for Biotechnology Information.

\section{Declarations}

\section{Declaration of Interest Statement}

We declare that we have no financial and personal relationships with other people or organizations that can inappropriately influence our work, there is no professional or other personal interest of any nature or kind in any product, service and/or company that could be construed as influencing the position presented in, or the review of, the manuscript entitled.

\section{Ethics approval and consent to participate}

Not applicable.

\section{Consent for publication}

Written informed consent for publication was obtained from all participants.

\section{Availability of supporting data}

Supporting data is available in the NCBI database. Raw data has been deposited in NCBI with the project accession PRJNA505902. BioSample accessions: SAMN10438768, SAMN10438757-60, SAMN10438763 and SAMN10438766.

\section{Competing interests}

The authors declare that they have no competing interests.

\section{Funding}

This project was supported by the key project of Science-technology basic condition platform from The Key Project of Science and Technology Program of Guizhou Province "Study of biological and ecological vales for Fanjingshan World Natural Heritage Nomination of Tongren City (3052 2015 Qiankehe SY)”, Science-technology basic condition platform from The Ministry of Science and Technology of the People's Republic of China (Grant 
No. 2005DKA21402), Provincial Governor Fund of Guizhou (Qian-Sheng-Zhuan-He-Zi (2010) 14) project and the Youth Innovation Promotion Association of Chinese Academy of Sciences (2011077).

\section{Author contributions}

ZL, XQ, HW, and LH, performed the experiments, XQ, and ZW, analyzed the data, ZL and HW, wrote the paper, and participated in the design of the study. $X Q, H W$, and $Z \mathrm{~W}$, participated in data statistics and the draft manuscript. $\mathrm{JZ}$, and ZL, conceived the study, participated in its design and coordination, and helped to draft the manuscript. All authors read and approved the final manuscript.

\section{Acknowledgements}

We are grateful to Kai Gao, Jian Chen, Zhonglong Cen and Gang Li for their help in sampling and laboratory work. We thank Martin Burrows and Wenhua Xiong for assistance in writing and data analysis.

\section{References}

[1] Zhu, S. (1989). The Loaches of the Subfamily Nemacheilinae in China (Cypriniformes: Cobitidae). Jiangsu Science \& Technology Publishing House.

[2] Liu, Z.J., Wen, H.M., Hailer, F., et al (2019). Pseudogenization of Mc1r gene associated with transcriptional changes related to melanogensis explains leucistic phenotypes in Oreonectes cavefish (Cypriniformes, Nemacheilidae. Journal of Zoological Systematics and Evolutionary Research, 57, 900-909.

[3] Deng, H., Xiao, N., Hou, X., et al (2016). A new species of the genus Oreonectes (Cypriniformes: Nemacheilidae) from Guizhou, China. Zootaxa, 4132, 143-150.

[4] Parra, G., Bradnam, K., \& Korf, I. (2007). CEGMA: a pipeline to accurately annotate core genes in eukaryotic genomes. Bioinformatics, 23, 1061-67.

[5] Simão, F.A., Waterhouse, R.M., loannidis, P., et al (2015). BUSCO: assessing genome assembly and annotation completeness with single-copy orthologs. Bioinformatics, 31, 3210-12.

[6] Cingolani, P., Platts, A., Wang le, L., et al (2012). A program for annotating and predictingthe effects of single nucleotide polymorphisms, SnpEff: SNPs in the genome of Drosophila melanogaster strain w1118; iso-2; iso-3., Fly, 6, 80-92.

[7] Huang da, W., Sherman, B.T., \& Lempicki, R.A. (2009). Systematic and integrative analysis of large gene lists using DAVID Bioinformatics Resources. Nature Protocols, 4, 44-57.

[8] Javier A. Rodriguez Robles , David A. Good , David B. Wake. Brief History of Herpetology in the Museum of Vertebrate Zoology, University of California, Berkeley, with a List of Type Specimens of Recent Amphibians and Reptiles:. 2003.

[9] Gaia Gestri, Robert J. Osborne, Alexander W. Wyatt, et al. Reduced TFAP2A function causes variable optic fissure closure and retinal defects and sensitizes eye development to mutations in other morphogenetic regulators. 2009, 126(6):791-803. 
[10] Li, H., \& Durbin, R. (2009). Fast and accurate short read alignment with Burrows-Wheeler transform. Bioinformatics, 25, 1754-60.

[11] Xu, H.B., Luo, X., Qian, J., et al (2012). FastUniq: A Fast De Novo Duplicates Removal Tool for Paired Short Reads. PLoS One, 7, e52249.

[12] Korf, I. (2004). Gene finding in novel genomes. BMC Bioinformatics, 5, 59.

[13] Walker, B.J., Abeel, T., Shea, T., et al (2014). et al. Pilon: an integrated tool for comprehensive microbial variant detection and genome assembly improvement. PLoS One, 9, e112963.

[14] Li, H., Handsaker, B., Wysoker, A., et al (2009). The sequence alignment/map format and SAMtools. Bioinformatics, 25, 2078-79.

[15] Xu, Z., \& Wang, H. (2007). LTR_FINDER: an efficient tool for the prediction of full-length LTR retrotransposons. Nucleic Acids Research, 35, W265-68.

[16] Han, Y., \& Wessler, S.R. (2010). MITE-Hunter: a program for discovering miniature inverted-repeat transposable elements from genomic sequences. Nucleic Acids Research, 38, e199.

[17] Price, A.L., Jones, N.C., \& Pevzner, P.A. (2005). De novo identification of repeat families in large genomes. Bioinformatics 21 Suppl, 1, i351-58.

[18] Edgar, R.C. (2004). MUSCLE: multiple sequence alignment with high accuracy and high throughput. Nucleic Acids Research, 32, 1792-97.

[19] Wicker, T., Sabot, F., Hua-Van, A., et al (2007). A unified classification system for eukaryotic transposable elements. Nature Reviews Genetics, 8, 973-82.

[20] Jurka, J., Kapitonov, V.V., Pavlicek, A., et al (2005). Repbase Update, a database of eukaryotic repetitive elements. Cytogenet Genome Research, 110, 462-67.

[21] Tarailograovac, M., \& Chen, N. (2004). Using RepeatMasker to identify repetitive elements in genomic sequences. Current Protocols in Bioinformatics Chapter, 4, Unit, 410.

[22] Burge, C., \& Karlin, S. (1997). Prediction of complete gene structures in human genomic DNA. Journal of Molecular Biology, 268, 78-94.

[23] Stanke, M., \& Waack, S. (2003). Gene prediction with a hidden Markov model and a new intron submodel. Bioinformatics 19 Suppl, 2, ii215-25.

[24] Majoros, W.H., Pertea, M., \& Salzberg, S.L. (2004). TigrScan and GlimmerHMM: two open source ab initio eukaryotic gene-finders. Bioinformatics, 20, 2878-79.

[25] Blanco, E., Parra, G., \& Guigo, R. (2007). Using geneid to identify genes. Current Protocols in Bioinformatics Chapter, 4, Unit, 43.

[26] Keilwagen, J., Wenk, M., \& Erickson, J.L. (2016). Schattat MH, Grau J, Hartung F. Using intron position conservation for homology-based gene prediction. Nucleic Acids Research, 44, e89. 
[27] Altschul SF, Gish W, Miller W et al (1990). Basic local alignment search tool. Journal of Molecular Biology. 1990; 215:403-10.

[28] Campbell, M.A., Haas, B.J., Hamilton, J.P., et al (2006). Comprehensive analysis of alternative splicing in rice and comparative analyses with Arabidopsis. BMC Genomics, 7, 327.

[29] Kim, D., Landmead, B., \& Salzberg, S.L. (2015). HISAT: a fast spliced aligner with low memory requirements. Nature Methods, 12, 357-60.

[30] Pertea, M., Pertea, G.M., Antonescu, C.M., et al (2015). StringTie enables improved reconstruction of a transcriptome from RNA-seq reads. Nature Biotechnology, 33, 290-95.

[31] Haas, B.J., Papanicolaou, A., Yassour, M., et al (2013). De novo transcript sequence reconstruction from RNAseq using the Trinity platform for reference generation and analysis. Nat Protoc, 8(8):1494-512.

[32] Tang, S., Lomsadze, A., \& Borodovsky, M. (2015). Identification of protein coding regions in RNA transcripts. Nucleic Acids Research, 43, 588-588.

[33] Haas, B.J., Salzberg, S.L., Zhu, W., et al (2008). Automated eukaryotic gene structure annotation using EVidenceModeler and the Program to Assemble Spliced Alignments. Genome Biology, 9, R7.

[34] Tatusov, R.L., Natale, D.A., Garkavtsev, I.V., et al (2001). The COG database: new developments in phylogenetic classification of proteins from complete genomes. Nucleic Acids Research, 29, 22-8.

[35] Kanehisa, M., \& Goto, S. (2000). KEGG: kyoto encyclopedia of genes and genomes. Nucleic Acids Research, 28, 27-30.

[36] Boeckmann, B., Bairoch, A., Apweiler, R., et al (2003). The SWISS-PROT protein knowledgebase and its supplement TrEMBL in 2003. Nucleic Acids Research, 31, 365-70.

[37] Conesa, A., Gotz, S., Garcia-Gomez, J.M., et al (2005). Blast2GO: a universal tool for annotation, visualization and analysis in functional genomics research. Bioinformatics, 21, 3674-76.

[38] Lowe, T.M., \& Eddy, S.R. (1997). tRNAscan-SE: a program for improved detection of transfer RNA genes in genomic sequence. Nucleic Acids Research, 25, 955-964.

[39] Nawrocki, E.P., \& Eddy, S.R. (2013). Infernal 1.1: 100-fold faster RNA homology searches. Bioinformatics, 29, 2933-35.

[40] Griffiths-Jones, S., Moxon, S., Marshall, M., Khanna, A., Eddy, S.R., \& Bateman, A. (2005). Rfam: annotating non-coding RNAs in complete genomes. Nucleic Acids Research, 33, 121-24.

[41] Griffiths-Jones, S., Grocock, R.J., van Dongen, S., Bateman, A., \& Enright, A.J. (2006). miRBase: microRNA sequences, targets and gene nomenclature. Nucleic Acids Research, 34, D140-44.

[42] Sayers, E.W., Agarwala, R., Bolton, E.E., et al (2018). Database resources of the National Center for Biotechnology Information. Nucleic Acids Research, 5. 
[43] Altschul, S.F., Madden, T.L., Schäffer, A.A., et al (1997). Gapped BLAST and PSI-BLAST: a new generation of protein database search programs. Nucleic Acids Research, 25, 3389-402.

[44] Fischer, S., Brunk, B.P., Chen, F., et al (2011). Using OrthoMCL to assign proteins to OrthoMCL-DB groups or to cluster proteomes into new ortholog groups. Current Protocols in Bioinformatics, 35, 6.12.1-19.

[45] De Bie, T., Cristianini, N., Demuth, J.P., \& Hahn, M.W. (2006). CAFE: a computational tool for the study of gene family evolution. Bioinformatics, 22, 1269-71.

[46] Li, H., \&Durbin, R., (2009). Fast and accurate short read alignment with Burrows Wheeler transform. Bioinformatics, 25, 1754-60.

[47] McKenna, A., Hanna, M., Banks, E., Sivachenko, A., et al (2010). The Genome Analysis Toolkit: a MapReduce framework for analyzing next-generation DNA sequencing data. Genome Research, 20, 1297-303.

[48] Stamatakis, A. (2014). RAxML version 8: a tool for phylogenetic analysis and post-analysis of large phylogenies. Bioinformatics 30, 1312-13.

[49] Harris RS. (2007). Improved pairwise alignment of genomic DNA. Doctoral dissertation. Pennsylvania State University University Park.

\section{Figures}



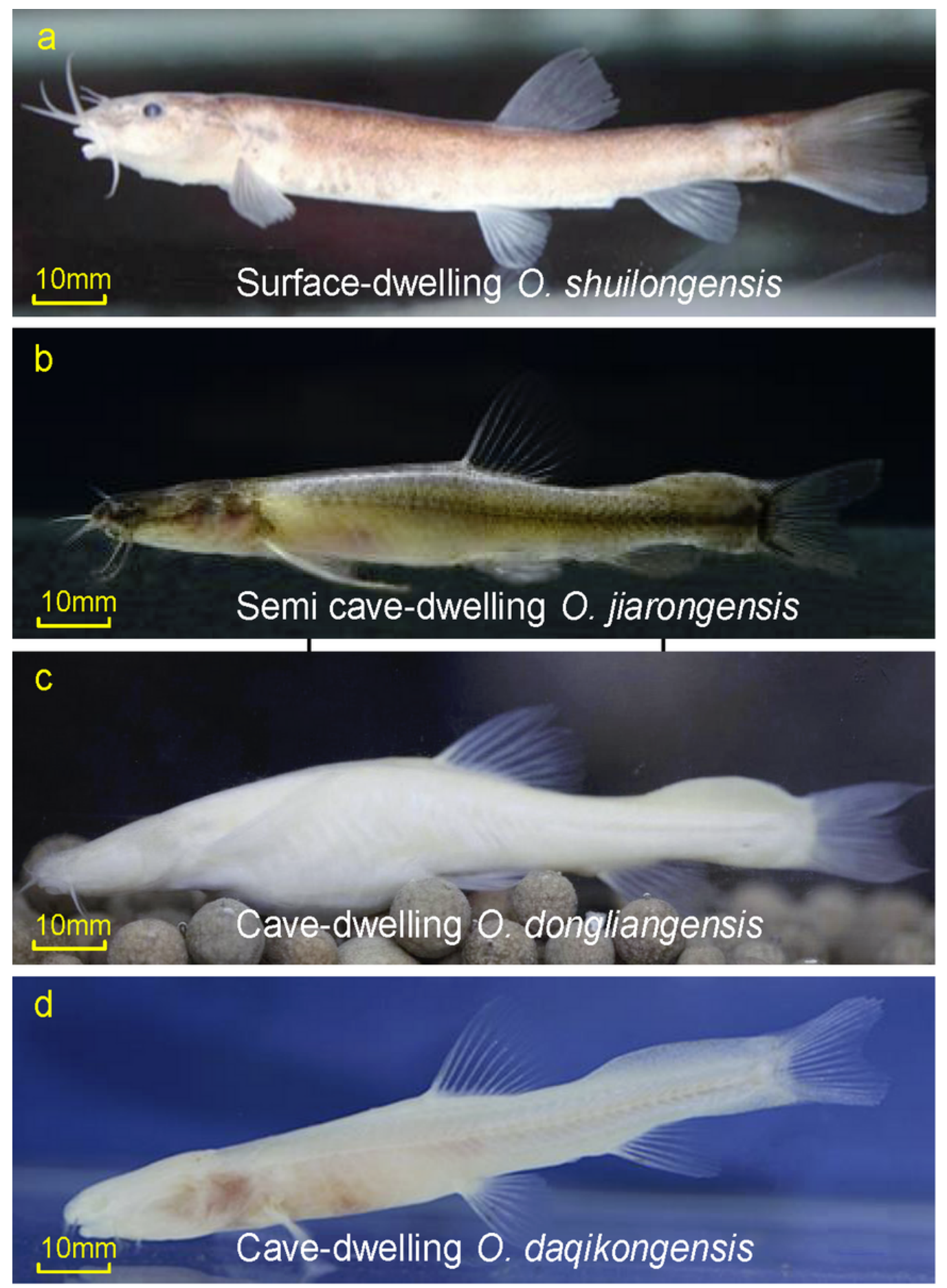

Figure 1

Photo of (a) O. shuilongensis, (b) O. jiarongensis, (c) O. dongliangensis and (d) O. daqikongensis. 


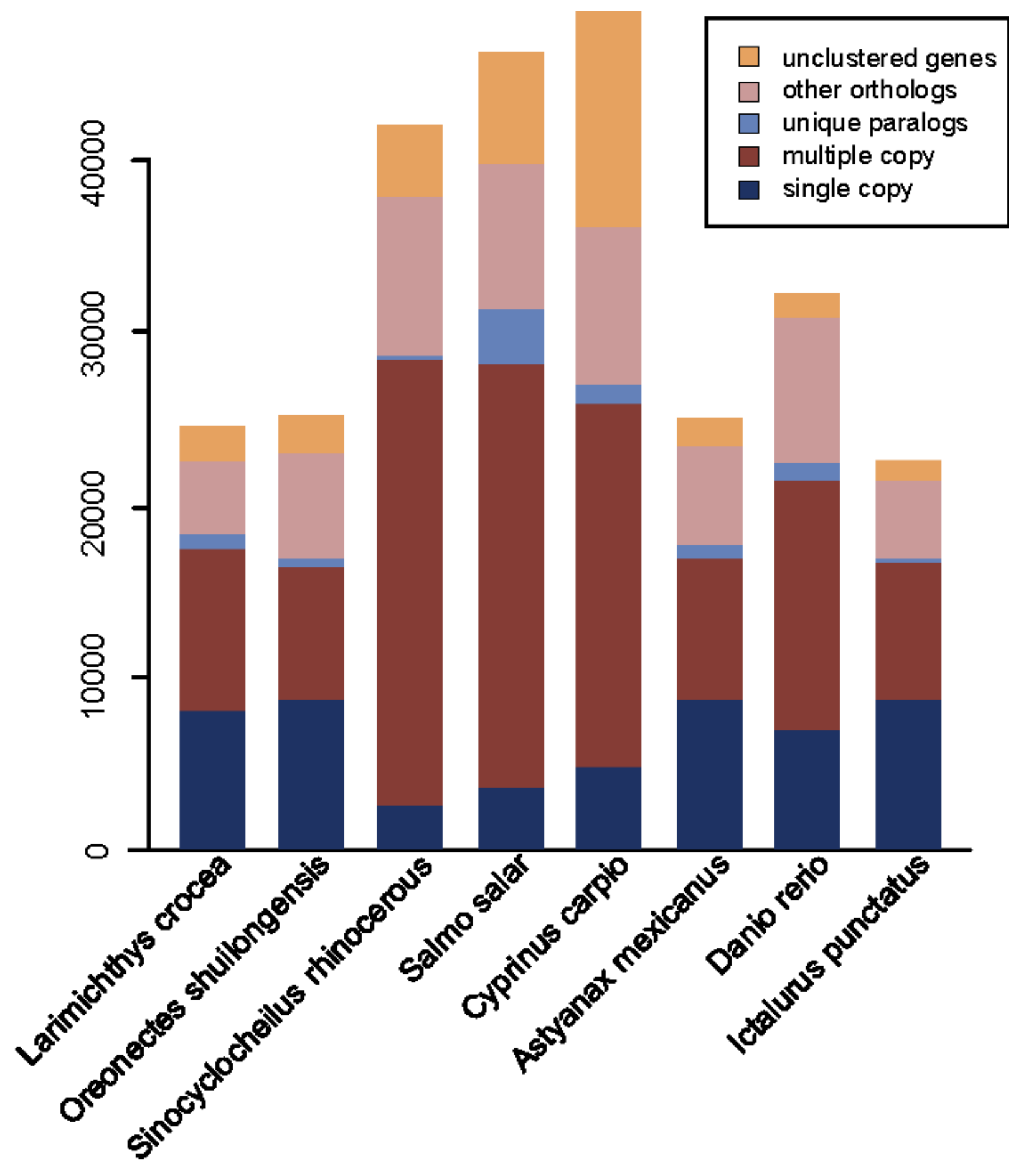

Figure 2

Gene family comparison between $\mathrm{O}$. shuilongensis and other fish species. The $\mathrm{Y}$-axis represented the gene number for each class: single-copy (one gene for each species), multiple-copy (more than one gene for each species), unique paralogs (no genes in other species), other orthologs (other cases in gene clusters) and un-clustered genes (genes that did not clustered with other genes). 

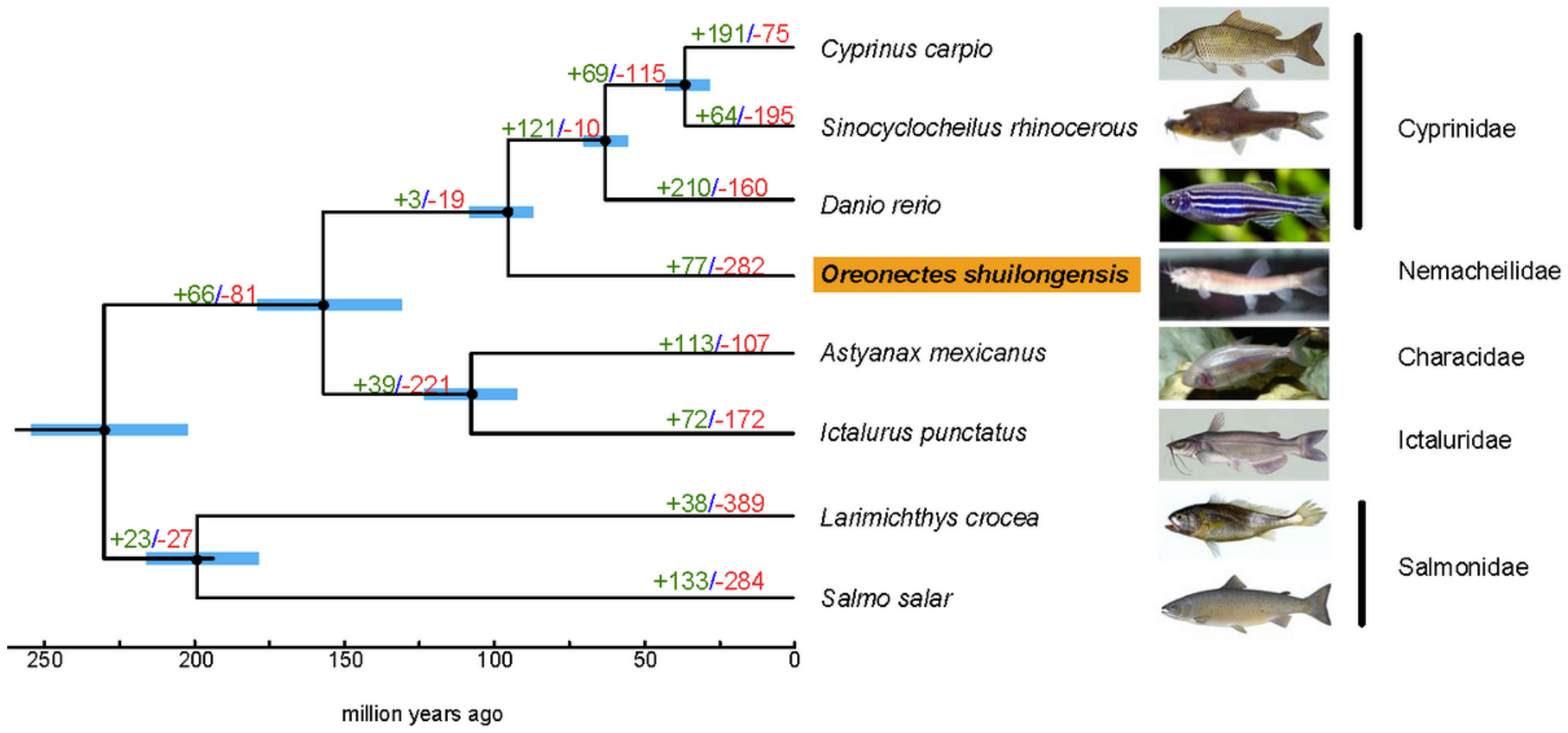

Figure 3

Phylogenetic relationships and estimated divergence times in million years ago (MYA) of 0 . shuilongensis and other fish. The numbers on each branch correspond to the numbers of gene families that have expanded (green) or contracted (red).

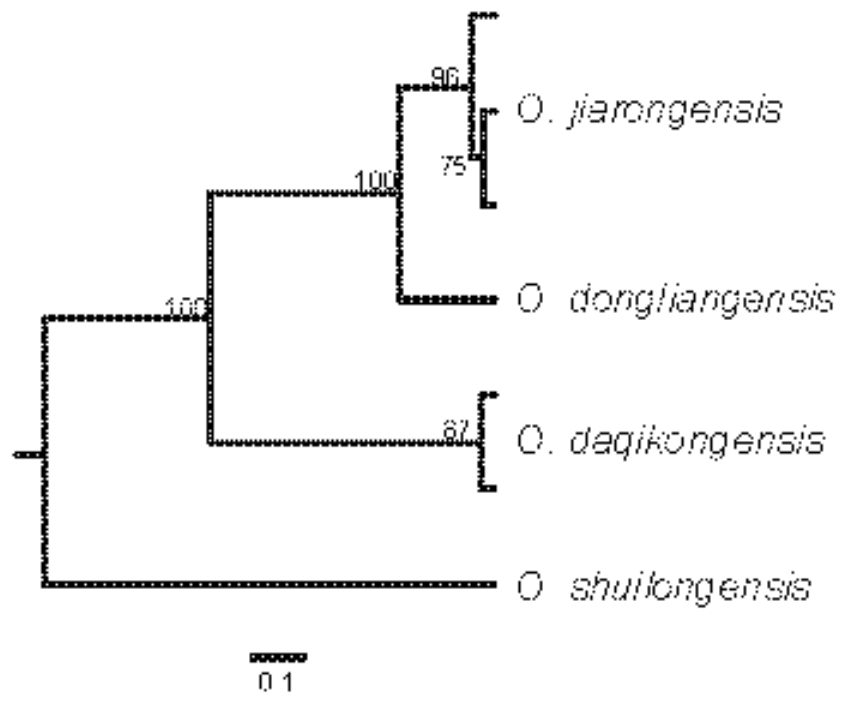

Figure 4

Neighbor-joining phylogenomic tree of Orenectus fishes, based on ML distances calculated from 1,364,638 SNPs. Numbers on branches denote support estimated from 1000 bootstrap replicates. The root was obtained from the orthologous sequences of Zebrafish. 

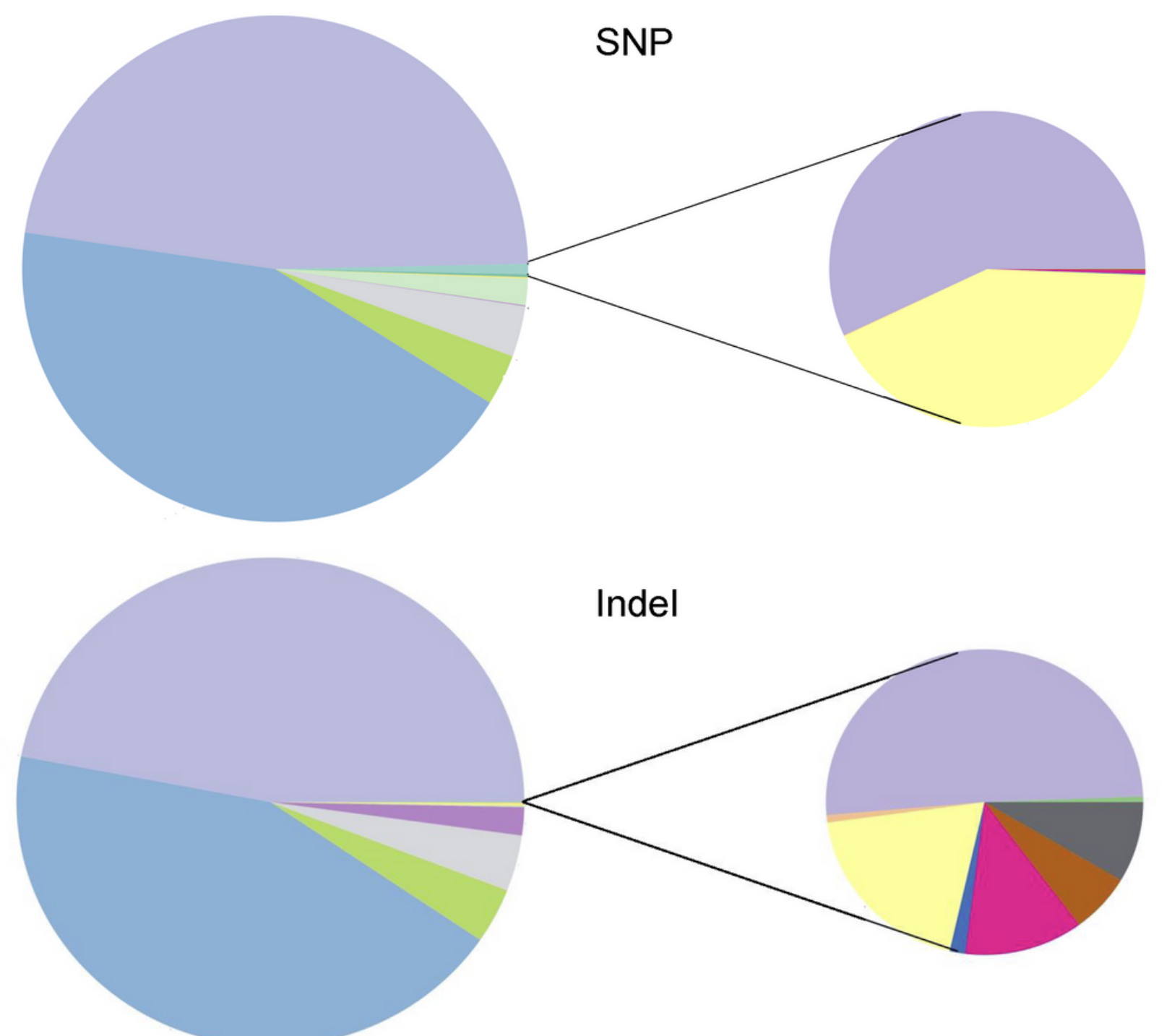

CDS

SPLICE_SITE_DONOR

Other

SPLICE_SITE_ACCEPTOR

INTERGENIC

START_GAINED

DOWNSTREAM

\section{SPLICE_SITE_REGION}

UPSTREAM

— UTR_5_PRIME

INTRON

INTRAGENIC

UTR_3_PRIME

NON_SYNONYMOUS_START
SYNONYMOUS_CODING

START_LOST NON_SYNONYMOUS_CODING

STOP_LOST

STOP_GAINED

SYNONYMOUS_STOP

\section{Figure 5}

SNPs (a) and Indels (b) annotation of cave-dwelling O. jiarongensis, O. daqikongensis and O. shuilongensis. 


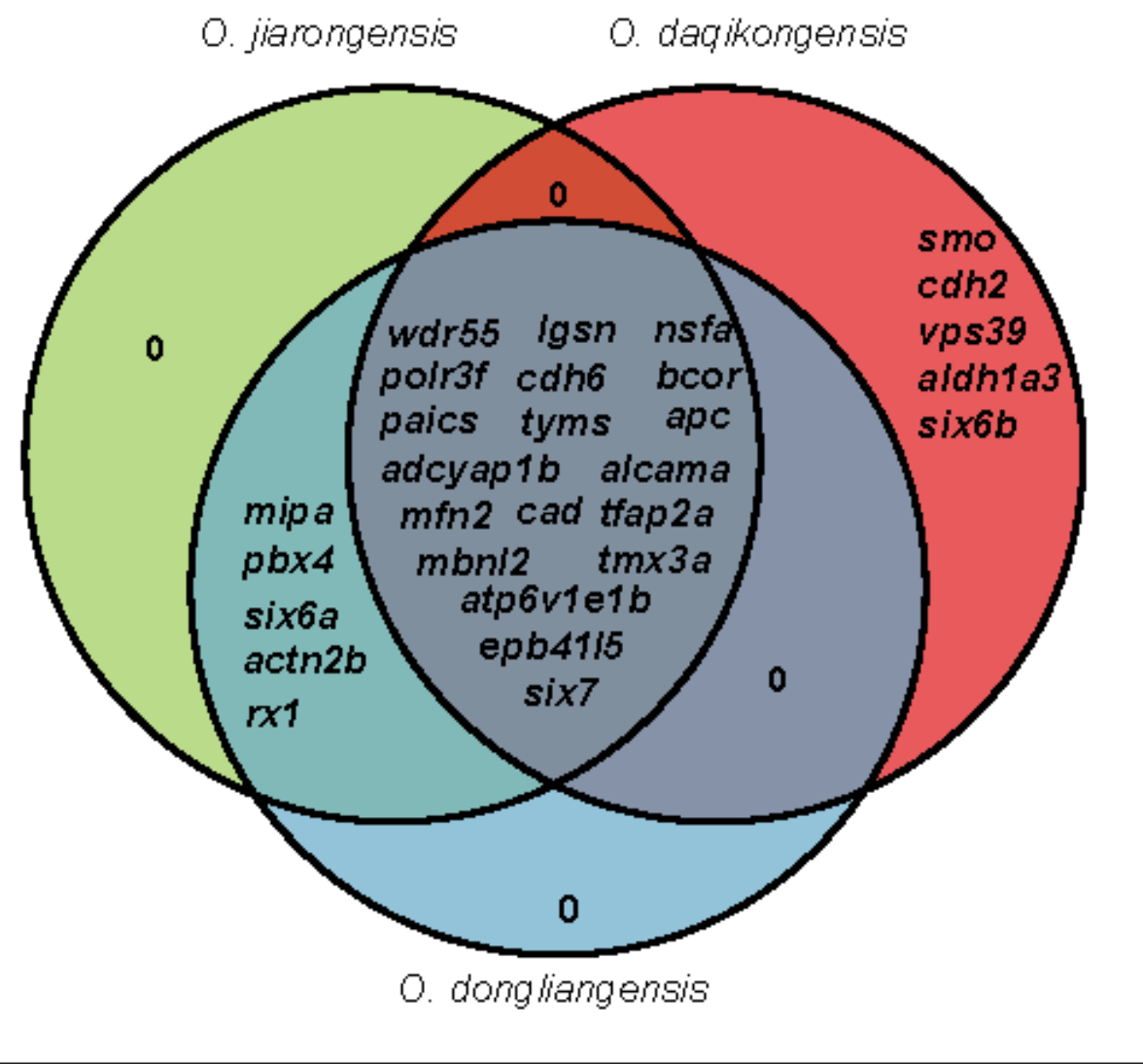

Figure 6

Pseudogenes related to the GO terms "retina development in camera-type eye" and "eye development" among cave-dwelling 0 . jiarongensis, 0 . daqikongensis and 0 . shuilongensis. 


\section{Deletion in 0 . daqikongensis}

\section{Cave-dwelling}

\section{0. shuilongensis \\ Surface-dwelling}

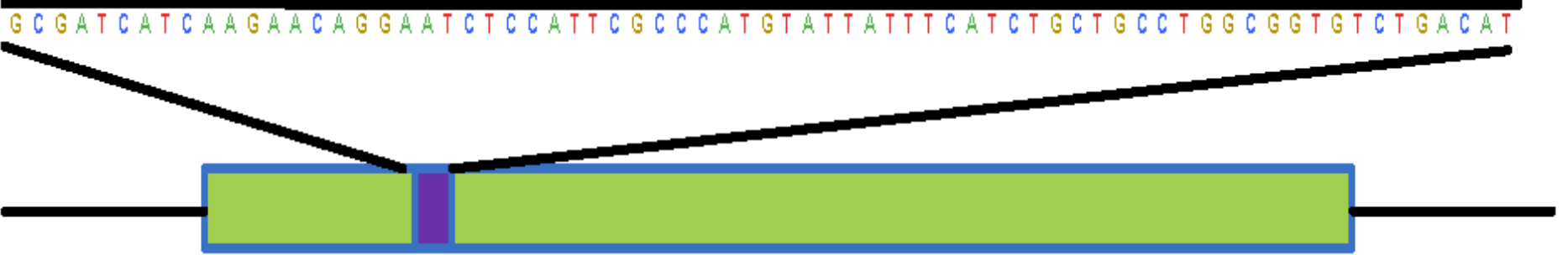

MC1R conding region

Figure 7

Pseudogene of MC1R in 0. daqikongenis caused by 29 bp deletion in coding region.

\section{Supplementary Files}

This is a list of supplementary files associated with this preprint. Click to download.

- ArriveChecklist.pdf

- cavefishgenomeSI.docx 
Page 23/23 